\title{
MAC specifications for a WPAN allowing both energy saving and guaranteed delay *
}

\author{
Part B: Optimization of the intra-star exchanges for \\ MaCARI
}

\author{
Erwan Livolant, Adrien van den Bossche, and Thierry Val \\ Université de Toulouse ; UTM ; LATTIS (LAboratoire Toulousain de Technologie et \\ d'Ingénierie des Systèmes) ; SCSF research group \\ IUT Blagnac, 1 place Georges Brassens ; BP60073 ; F-31703 Blagnac, France \\ erwan.livolant@laposte.net, vandenbo@iut-blagnac.fr, val@iut-blagnac.fr
}

\begin{abstract}
Industrials have been increasingly interested in sensor and actuator networks to monitor and control their installations. The recent IEEE 802.15.4 standard has been developed to address vital issues of these networks, such as limited battery power and low processing capabilities. However, the standard does not meet all the requirements of industrial networks. For example, only some of the IEEE 802.15.4 nodes save energy, and the delay for a sensor to activate an actuator is not bounded. Our research on energy-efficient MAC protocol is divided into two parts: Part A consists in introducing a flexible, synchronized treebased MAC protocol called MaCARI and Part B deals with optimizations that can be performed within each star.

This paper focuses on Part B, that is, on the intra-star MaCARI protocol. Our proposal is an incremental protocol with different options which increase respectively the previous one in terms of bandwidth and energy saving. A hardware prototyping of the last option has been done in order to validate our proposal.
\end{abstract}

Keywords: wireless sensor networks, IEEE 802.15.4, energy efficiency, QoS, MAC layer.

\section{Introduction}

The OCARI project [2] aims to optimize the wireless communications for industrial networks with sensors and actuators. One of the challenges of the project is the proposition of a new Medium Access Control (MAC) layer. The physical layer used is the IEEE 802.15.4 PHY2450 [3] also used for ZigBee networks [4]. OCARI network general topology is an ad-hoc mesh network, made of two types of devices: coordinators (routers, i.e. Full Function Devices, or FFD, in

\footnotetext{
* The OCARI project is a partnership between EDF R\&D, DCNS, INRIA, LRI, LIMOS, One RF Technology and LATTIS with the support of the ANR (French National Research Agency) [1]. LIMOS and LATTIS are in charge of the MaCARI medium access control development.
} 
the IEEE 802.15.4 terminology) and end-devices (sensors, i.e. Reduced Function Devices, or RFD, in the IEEE 802.15.4 terminology). As in the 802.15.4 standard, an end-device must be associated to an unique coordinator. A coordinator and its end-devices form a star topology. However, the coordinators are free to communicate with others coordinators if they are in range with each other (peer-to-peer topology). The general MAC-layer proposed by the OCARI project is named MaCARI (MAC for oCARI) [5]. MaCARI relies on a beaconed tree-based mechanisms between coordinators. This MAC layer organizes the device synchronization and the waking up/transmitting/receiving scheduling. The temporal organization is divided into three parts [5] (Fig. 1).

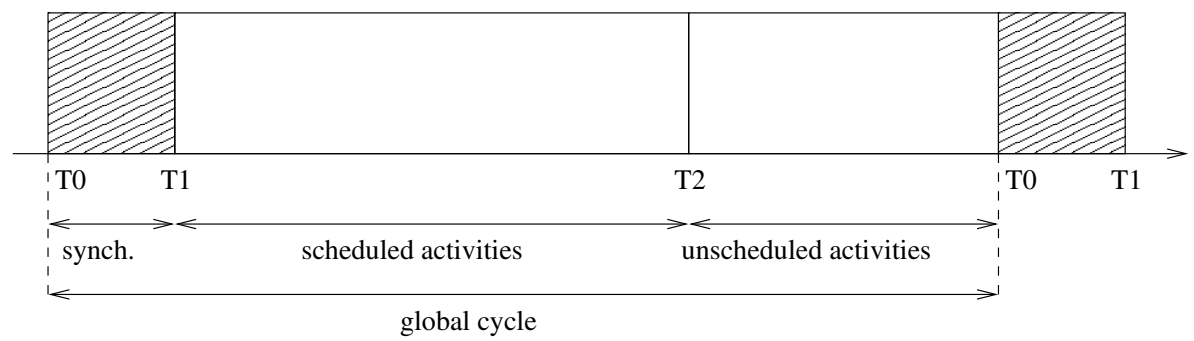

Fig. 1. Global cycle with its three periods.

- A first period, named synchronization period $\left(T_{0}-T_{1}\right)$, broadcasts the synchronization and the scheduling over the entire network.

- A second period, named scheduled activities period $\left(T_{1}-T_{2}\right)$, is dedicated to the tree-scheduled communications. In this period, each star has a timeslot where no other star can transmit to strictly avoid collisions. The only devices authorized to transmit are the devices of the concerned star (coordinator, end-devices) plus the father coordinator of the star (tree topology). The present article deals with the intra-star organization of this period.

- A third period, named unscheduled activities period $\left(T_{2}-T_{0}\right)$, where free communication are possible by using CSMA/CA protocol for instance. In this period, communications may not respect the tree topology. Devices can communicate as soon as they are in the same radio range. The routing protocol proposed by the network-layer uses this period.

\section{OCARI basic hypothesis}

This section reminds the principal hypothesis of the OCARI project. These hypotheses induce some constraints to MaCARI and have a direct impact on directions and optimizations proposed. 
- Each coordinator manages the activity period of its star during the dedicated period it gets between $T_{1}$ and $T_{2}$. All end-devices are awaken and are waiting for synchronization adjustment. Once the working period has passed, devices may enter in doze mode, as detailed in the next sections.

- The PAN coordinator schedules the use of the medium for each star. This slot enables intra-star communications without collision since the medium is dedicated to a single star during the slot.

- During this period, we suppose that the only star authorized to transmit data is the star which receives the PAN coordinator authorization. In fact, we suppose that collisions are not possible with the others stars.

- Basically there are no direct communications possible between two enddevices. End-devices can communicate with their coordinator thanks to the indirect data transfer described by IEEE 802.15.4 standard [3]. We will see in the next sections that this point can be improved in intra-star MaCARI to optimize throughput.

- The OCARI project assumes a single transmit (TX) power level. A preliminary study [6] has shown that IEEE 802.15.4 transceivers do not save a lot of energy by reducing TX-power (unlike WiFi, WiMAX or GSM transceivers).

- Two same star end-devices may not be in radio range with each others. Of course, a star-coordinator must be in range of all its end-devices.

- Moreover, a star-coordinator may know all its end-devices: the OCARI network is not a spontaneous self-organized network since the application imposes that each sensor is logically "linked" to a particular coordinator. In the OCARI aimed applications, sensors are previously defined and located by the application-level.

- The propagation conditions are quiet stable.

- At last, we consider that devices (end-devices and coordinators) are not mobile. The OCARI network does not permit mobility. At best, end-devices may be mobile but stay in the star, since the protocol does not provide for any handover mechanism.

\section{Intra-star MaCARI}

Our protocol proposition defines the MAC protocol inside the star when the FFD (Full Function Device) is able to communicate by a beacon reception. During $\left(T_{1}-T_{2}\right)$ (Sect. 1), the star owns a guaranteed medium access without collision or interference risks against the other nodes of the OCARI network.

\subsection{An incremental protocol: intra-star MaCARI options}

We propose several MAC options (Fig. 2), from the simplest to the more complex, as an incremental protocol depending on:

- Comparative performance analysis of these options (by simulation and/or prototype metrology). 
- Maximum end-device number by star: indeed, a complex intra-star MaCARI method could be useless if the star owns only a couple of end-devices.

- Quantity and kind of exchanged data: as previously, a complex intra-star MaCARI method could be useless if each end-device only transmits a short frame by minute. On the other hand, if end-devices transmit longer frames with timed-constrained data, with a stronger periodicity, a MaCARI with QoS is required. Such a method, deterministic and with QoS, could enhance the energy saving issue.

- Prototype implementation feasibility and device architecture chose (type of microcontroller(s), memory size, data exchange interfaces, etc.)

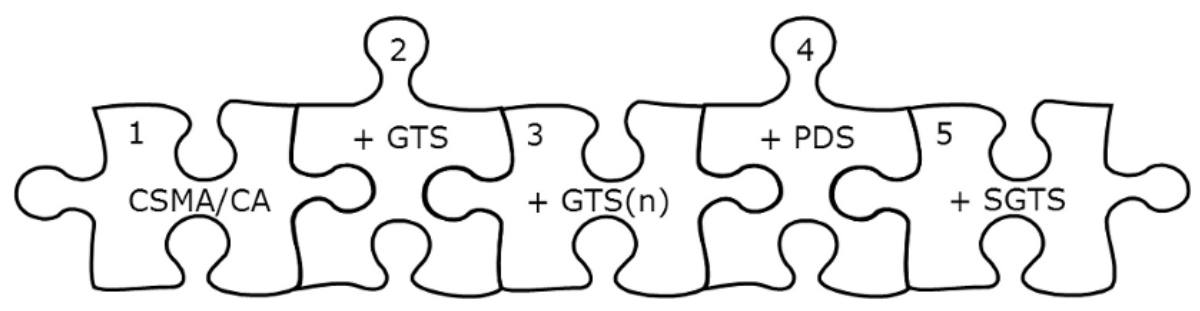

Fig. 2. Incremental MAC options.

\subsection{Full slotted CSMA/CA beaconed by the FFD}

The simplest intra-star method could be the slotted CSMA/CA one. Every communication is beaconed between $T_{1}$ and $T_{2}$ during this kind of superframe (as in IEEE 802.15.4 vocabulary). Each node uses the same distributed protocol. As an end-device, a node can only exchange with its FFD. Data for end-devices are transmitted by the FFD during a timeslot requested by the end-device. Concurrent accesses are avoided by the CSMA/CA method. This best effort MAC is relevant for a few-loaded star in regards of the QoS requirements.

\subsection{CSMA/CA + GTS (Guaranteed Time Slot)}

The random characteristic of the CSMA/CA implies a collision risk between two or more transceivers. In fact, quasi-simultaneous CCAs (Clear Channel Assessment) function calls by concurrent transceivers entail a collision. Without acknowledgment response because of the data collision, each node uses a random backoff time which could be unfortunately the same. In consequences, a new collision might appear. This phenomenon has a small but non-null probability to continue endlessly. The consequences of this could be insignificant for most applications but could be disastrous when the submitted network load is important. Indeed, the more frames to be transmitted, the more collisions, and the 
more frames to be retransmitted. A collapse phenomenon occurs which implies a quasi-null efficient bandwidth. Delays are also increased. This fact could be unacceptable for time-constrained applications.

This problem could be avoided by using, in each star, a specific access method using a superframe with two periods: CAP (Contention Access Period) and CFP (Contention Free Period). CAP uses only CSMA/CA. This period permits a basic QoS for non-priority flow without temporal guarantees. This best effort algorithm can be used for sporadic and unexpected flows. CFP uses dedicated timeslots allocated by the FFD to its end-devices. These slots are named GTS (Guaranteed Time Slot).

According to the application needs of each sensor, the end-device requests to its coordinator a GTS allocation for the next superframe. If the minimal size of the CAP is not reached, the FFD can allocate a new GTS. The CAP is reduced accordingly. If an end-device does not use its GTS during a specific duration, this GTS is automatically suppressed (timeout). The end-device must request a new GTS attribution for next superframes. The IEEE 802.15.4 standard has proposed this algorithm, but it is rarely implemented on commercial devices.

Two QoS levels for two different flow types are proposed. The first one for time-constrained flows is associated with known needs. The second one for best effort flows is associated with sporadic and unexpected data.

\subsection{CSMA/CA + GTS with multiple reservation levels}

The previous solution is an interesting option but its major drawback is the GTS static allocation for each superframe. For example, one GTS by superframe could be useless for a simple temperature sensor. It would be interesting to propose a service differentiation according to communication needs of end-device application. This intra-star MaCARI option proposition is based on special reservation level named 'n'. A GTS(n) is dedicated to an end-device according to its periodicity request:

- when $\mathrm{n}=0$ : for each superframe (as in IEEE 802.15.4 standard),

- when $n=1$ : every two superframes,

- in the general case: every $2^{n}$ superframes.

The principal advantage is the cohabitation of different guaranteed traffics according to different sensors. It is also possible to choose a reverse allocation principle. In a superframe a sensor could have more than one GTS. In this case, the reservation level is $n=1$. However, the end-device must request several GTSs in each superframe. The bandwidth allocated to such an end-device is increased.

The other advantage of this option is the power saving offered by this MAC layer. A sensor can commute to doze mode, especially when this sensor is not concerned by these superframes. If a temperature sensor has a high inertia, its end-device can wake up only every 4 or 8 superframes for instance for a fast and short temperature transmission. After this activity this end-device commute in battery saving mode. In the classical IEEE 802.15.4 protocol, a wake up is 
mandatory every superframe for using a GTS. In our proposition, it is possible to save timeslots with an optimized allocation only when it is necessary. This option maximizes doze mode outside GTSs.

\subsection{CSMA/CA + GTS(n) + PDS (Previously Dedicated Slot)}

The principal drawback of the previous option (also in the IEEE 802.15.4) is related to the GTS request which is necessary done by the end-device in CSMA/CA mode. This request mode is not fully deterministic because this random access is non-guaranteed to obtain GTS which is guaranteed. For sensible sensor applications that need guaranteed access, we propose a new intra-star MaCARI option based on PDS (Previously Dedicated Slot). A PDS is allocated to each specific sensor that uses this high level of QoS. A PDS is in fact an preallocated GTS. This PDS can be used by an end-device to transmit periodic data with high QoS. The end-device can also use this PDS to request more or less GTSs. This dynamic GTS allocation permits variation according to bandwidth and delay needs.

To avoid an overload of the used bandwidth, a large ' $n$ ' is associated to this first PDS. A performance analysis [6,7] shows that the bandwidth used for this PDS is very short $(0.78 \%$ for a PDS with $n=3)$.

\subsection{CSMA/CA + SGTS (Simultaneous GTS)}

The last proposed option permits a large-scale OCARI network. In fact, if the network is composed of a large number of devices, particularly for the intra-star part, the congestion is highly probable if the sensors are numerous. The star topology entails a bottleneck inside the range of the coordinator where every data flows converge from end-devices. In some applications, two end-devices could have to exchange data with each other. In this case, a centralize communication via the coordinator can be damageable for the star bandwidth. Typically, a sensor could directly send data to an actuator in the same star, and the actuator could send back an acknowledgment. Both end-devices must be at radio range with each other. This principle optimizes the end-to-end delay and reduces the load of the coordinator.

Moreover, multiple simultaneous communications between end-device pairs in the same star are conceivable under specific radio propagation conditions. Received signal strength of pairs must be different enough in order for a receiver to focus on its associated flow. The SGTS concept [8] consists in allocating the same timeslot to multiple end-device pairs (simultaneous medium access without collisions) (Fig. 3).

- (A) SGTS between end-device pairs

- (B) SGTS between [the coordinator and an end-device] and [an end-device pair]

SGTS feasibility conditions are based on: 


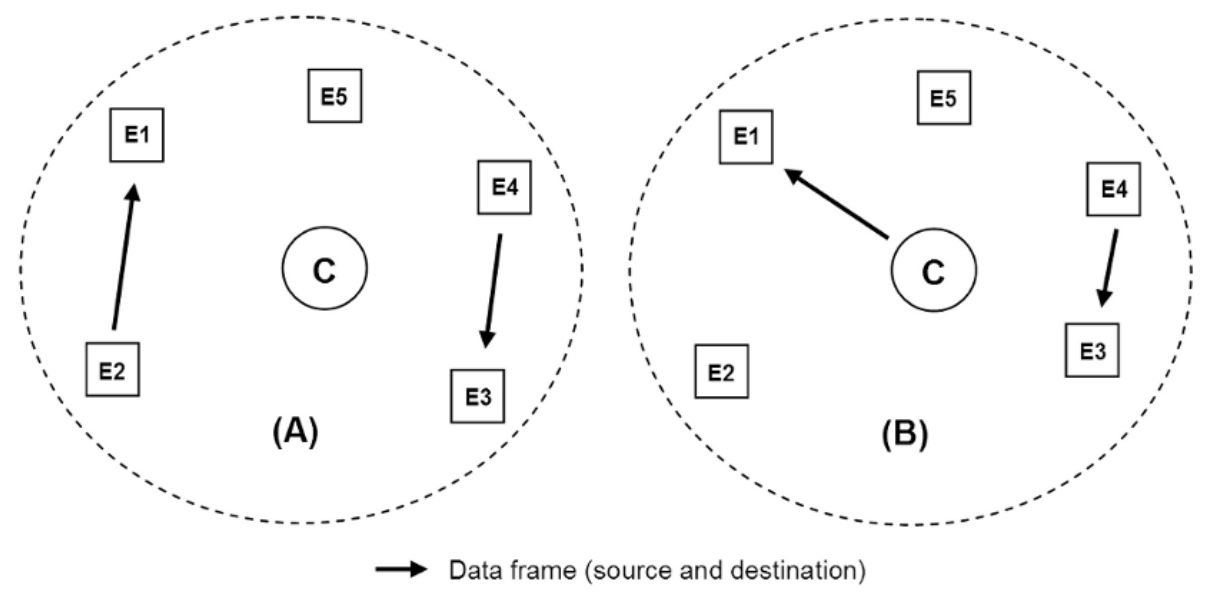

Fig. 3. SGTS concept.

- A measure of received signal strength from each emitter. This measure must be done in real time for every node which wants to using SGTS.

- A bilateral negotiation between nodes that want a simultaneous timeslot.

- A certain stability of propagation conditions.

For example (Fig. 3(A)) when E2 and E4 transmit data simultaneously, the end-device E1 measures that the signal strength of E4 is lesser than the signal strength of E2 with an additional margin. The coordinator (C) centralizes SGTSrelated data and organizes SGTS allocations to its end-devices.

The coordinator can be involved in a pair which negotiates a SGTS allocation. In Fig. 3(B), C communicates with E1 while E4 communicates with E3. In other words, a SGTS is allocated to $\mathrm{C} \Rightarrow \mathrm{E} 1$ while an other SGTS is allocated to $\mathrm{E} 4 \Rightarrow \mathrm{E} 3$, only if radio conditions are satisfied (as described previously).

As a parameter, the signal strength can be adjusted to increase the number of potential SGTS. In Fig. 4, E4 reduces its signal strength in order to reach only its nearest neighbor E3. In this case, SGTS between $\mathrm{E} 1 \Rightarrow \mathrm{C}$ and between $\mathrm{E} 4 \Rightarrow \mathrm{E} 3$ are possible.

The SGTS concept can be used for data frames without acknowledgments. In the case of acknowledgment requirements, acknowledgments must apply the same signal strength measurement and SGTS negotiation procedures. Thus, two different methods can be used:

- Accept a SGTS only if propagation conditions are symmetric.

- Acknowledge data in a specific field of an other data frame associated to a later SGTS.

A theoretical and metrological study on propagation conditions proved an acceptable reception threshold for SGTSs without collision risks. A margin must be respected to increase SGTS quality. 


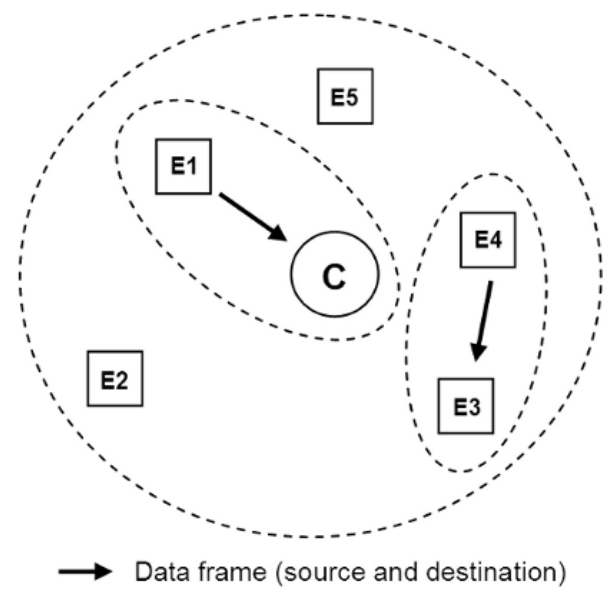

Fig. 4. SGTS with a receiver FFD.

\section{Preliminary study for SGTS concept validation}

The concept of SGTS needs to be validated and the non-perturbation margin has to be identified. The best way to validate this concept is the real hardware prototyping. Therefore we have developed a prototype of a simple MAC-layer and deployed a network based on a couple of Freescale IEEE 802.15.4 devices [9]. This type of 802.15.4 devices is totally reprogrammable, which allowed us to implement the SGTS functionality. This prototype also enables us to evaluate the non-perturbation margin evoked in Sect. 3.6.

\subsection{The prototype network and its topology}

The prototype network is composed of five nodes: a star-coordinator $(\mathrm{C})$ and four end-devices associated (synchronized) to this coordinator. The network topology and the superframe structure (frame scheduling) are respectively shown on Fig. 5 and Fig. 6.

For this case study, we consider that both E1 and E3 have obtained a GTS and can freely use it to send data to another end-device (respectively to E2 in slot \#2 and to E4 in slot \#3). Slot \#4 is used by both E1 and E3. In this study, the objective is to evaluate the non-perturbation margin, i.e. to measure the number of collisions in slot \#5. In order to evaluate the perturbation of the other transmitter (E3 for E2 and E1 for E4), each coordinator listens to the messages sent by the two nodes and gets the RSSI value during the slots \#2 and \#3. The RSSI difference is calculated at the end of slot \#3, only if both messages from E1 and E3 have been received. In slot \#4, the receivers E2 and E4 listens to the medium. If they receive the expected message, the result is positive. If they receive the message of the other device or if a collision is detected, the result is negative. 


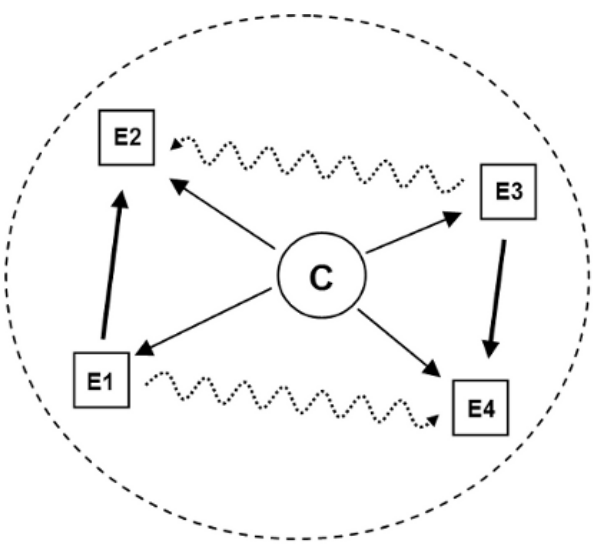

$\longrightarrow$ Beacon (synchronization)

$\longrightarrow$ Data frame (source and destination)

Received perturbation

Fig. 5. Prototype topology.

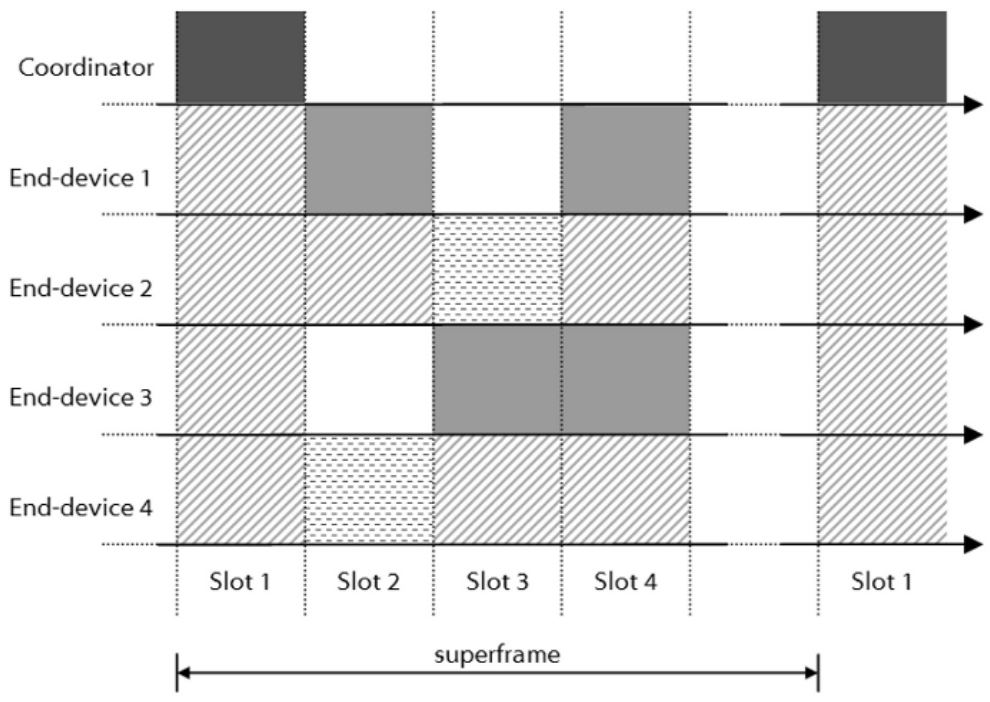

Fig. 6. Message temporal scheduling. 
Note that on the devices used for this study, the transmit power can be adjusted from $-16 \mathrm{dBm}$ to $+3.6 \mathrm{dBm}$. This functionality enables us to implement an automatic variation of the node transmitting power to increase the measure range without moving the devices. Measures have been realized both into an anechoic chamber (i.e. without any noise) and in a regular environment (i.e. with noise).

\subsection{Obtained results}

The results showed on Fig. 7 prove that the SGTS concept works correctly on a real prototype: two transmissions can be done at the same time without interfering the other receiver. The signal strength margin is small. In fact, the obtained results show that there is only a $6-7 \mathrm{~dB}$ window where the SGTS may not be envisaged because of an important risk of collision. We can considerate that a margin of $10 \mathrm{~dB}$ is safe.

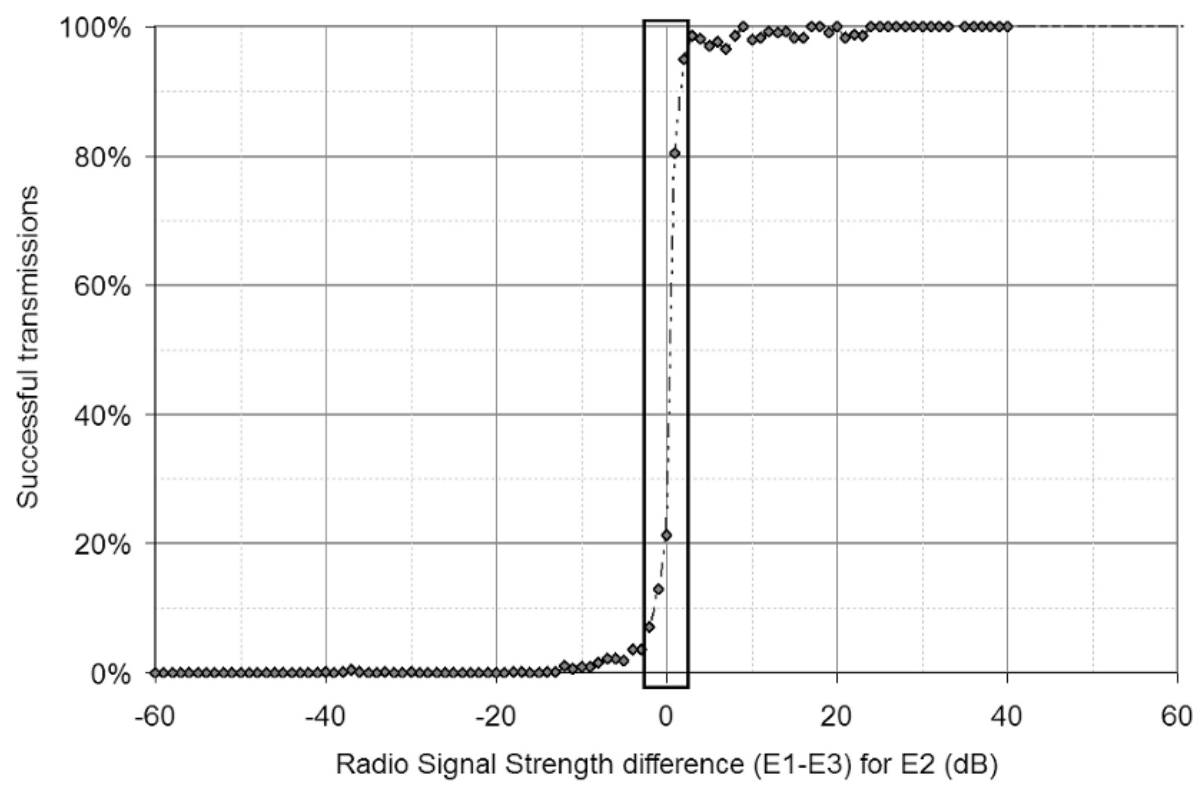

Fig. 7. Obtained results with the SGTS study in regular environment.

\section{Conclusion}

MaCARI proposes a core based on beaconed slotted CSMA/CA and allows several optional functionalities. The Figure 8 summarizes the five proposed options 
with their advantages and drawbacks. We plan to analyze the performance of these additional functionalities in a real prototype and consider the results as a reference. The option \#2 (IEEE 802.15.4 GTSs) may propose a first level of determinism. Unfortunately, there is no commercial implementation of the GTS. This specific part has to be implemented first. The option \#3, which requires the option \#2, proposes a higher QoS flexibility thanks to a mechanism of service differentiation. Moreover, the energy-saving resulting of this option was previously required by the OCARI project which aims to propose a MAClayer with energy-saving functionalities. Option \#4 which is based on PDS also requires options \#2 and \#3. It enables the higher level of determinism. Option \#5 plans to optimize the medium utilization between the network entities. In fact, the $250 \mathrm{kbps}$ has to be shared in the best way. This last option may be implemented without option \#2, \#3 and \#4. Nevertheless the SGTS concept is very interesting if it is used together with GTS(n) and PDS.

\begin{tabular}{|c|c|c|c|}
\hline & $\begin{array}{c}\text { IEEE } \\
\text { standard }\end{array}$ & $\begin{array}{c}\text { Our } \\
\text { Contribution }\end{array}$ & Comments \\
\hline (1) CSMA/CA & $\checkmark$ & & Simplest but only best effort \\
\hline (2) CSMA/CA + GTS & $\checkmark$ & & QoS but generally unimplemented \\
\hline (3) CSMA/CA + GTS(n) & & $\checkmark$ & Service Differentiation \\
\hline (4) CSMA/CA + GTS(n) + PDS & & $\checkmark$ & Fully deterministic \\
\hline (5) CSMA/CA + SGTS & & $\checkmark$ & Bandwidth optimization \\
\hline
\end{tabular}

Fig. 8. Summary of the MAC options.

\section{References}

1. ANR: The ANR web site http://www.agence-nationalerecherche.fr/documents/aap/2006/finance/Telecom-2006-resumes.pdf.

2. The OCARI project: The OCARI project web site http://ocari.lri.fr.

3. IEEE 802.15: Part 15.4: Wireless medium access control (MAC) and physical layer (PHY) specifications for low-rate wireless personal area networks (WPANs). Standard 802.15.4 R2006, ANSI/IEEE (2006)

4. Zigbee Alliance: Zigbee Specification. Standard Zigbee R2005 (2005)

5. Chalhoub, G., Guitton, A., Misson, M.: MAC specifications for a WPAN allowing both energy saving and guaranted delay - Part A: MaCARI: a synchronized treebased mac protocol. In: Submitted to WSAN 2008

6. van den Bossche, A., Val, T., Campo, E.: Prototyping and performance analysis of a QoS MAC-layer for industrial wireless network. In: FET2007 (7th IFAC International Conference on Fieldbuses and nETworks in industrial and embedded systems). (2007) 
7. van den Bossche, A.: Proposition d'une nouvelle méthode d'accès déterministe pour un réseau personnel sans fil à fortes contraintes temporelles. $\mathrm{PhD}$ thesis, Université de Toulouse (2007) In french.

8. van den Bossche, A., Val, T., Campo, E.: Proposition and validation of an original MAC layer with simultaneous medium accesses for low latency wireless control/command applications. In: IFAC WC08 (17th IFAC World Congress). (2008)

9. Freescale Semiconductor: Zigbee / IEEE 802.15.4 Freescale solution. Technology General Information (2004) 